Yervant Terzian, Daniel Weedman, Edward Khachikian, eds.

\title{
An Analysis of 900 Optical Rotation Curves
}

\author{
D. F. Roscoe
}

School of Mathematics, Sheffield University, Sheffield, S3 7RH, UK.

\begin{abstract}
Persic \& Salucci (1995; hereafter, PS) reduced raw $H_{\alpha}$ data from a large sample of southern sky spirals obtained by Mathewson, Ford \& Buchhorn (1992), to present $900 H_{\alpha}$ rotation curves. It is found that a power-law, $V_{\text {rot }}=A R^{\alpha}$, imposes extremely detailed correlations between the kinematic and the luminous properties of the galaxies through the model parameters $(A, \alpha)$; this law can be inverted to give a form of super Tulley-Fisher relationship for the determination of absolute magnitudes in terms of velocity information. Furthermore, there is very strong evidence suggesting the existence of discrete kinematic states for rotation curves (RCs hereafter) - which ultimately implies the luminosity evolution of galaxies to be similarly constrained to occupy discrete states.
\end{abstract}

\section{Introduction}

This analysis is based on the hypothesis that RCs in optical discs (away from the dynamical effects of the bulge) can be reasonably described by the law $V=A R^{\alpha}$, where $R>R_{\min }$ and $R_{\min }$ represents an estimate of the transition radius from bulge-dominated to disc-dominated dynamics. This latter estimate arises from the following process: if the innermost data point on any given $\mathrm{RC}$ is classified (by the regression software) as statistically 'unusual' in relation to the remaining data on the $\mathrm{RC}$, then it is assumed to be part of the bulge-dominated dynamical regime, and so is deleted. The process is applied iteratively until an innermost point is defined which is not classified as statistically unusual.

$V=A R^{\alpha}$ is then shown to provide an extremely detailed statistical resolution of kinematic data in terms of luminosity data. It is subsequently found that $\ln A$ appears to favour certain discrete values; given the detailed correlation between kinematic properties and luminosity properties, the final conclusion is that it is the luminosity evolution of galaxies which is ultimately constrained to occupy discrete states, implying a large-scale cosmic coherence phenomenon.

\section{A Necessary Condition For The Power-Law Hypothesis}

Given $V=A R^{\alpha}$ then, excepting for inevitable noise, a plot of any $\mathrm{RC}$ in the $(\log R, \log V)$ plane would lie on a straight line. Since RCs differ, for the 900 $\mathrm{RCs}$, we would obtain 900 different straight-line plots in the $(\log R, \log V)$ plane. Since the mean plot of 900 different plots of this type must also be a straight line, it follows that a necessary condition for optical RC data to be described 
by $V=A R^{\alpha}$ is that the mean plot of all the RCs in the $(\log R, \log V)$ plane must be a (statistically) straight line. So, the strategy of the initial analysis is described as follows:

- Reduce all the data in the combined sample of $900 \mathrm{RCs}$ to a uniform linear scale based on Tulley-Fisher (TF hereafter) distances; Superimpose all the data of the combined sample into a single data set; Divide the data set into bins of (convenient) width $0.057 \mathrm{kpc}$; Form the average of the data in each bin and plot it.
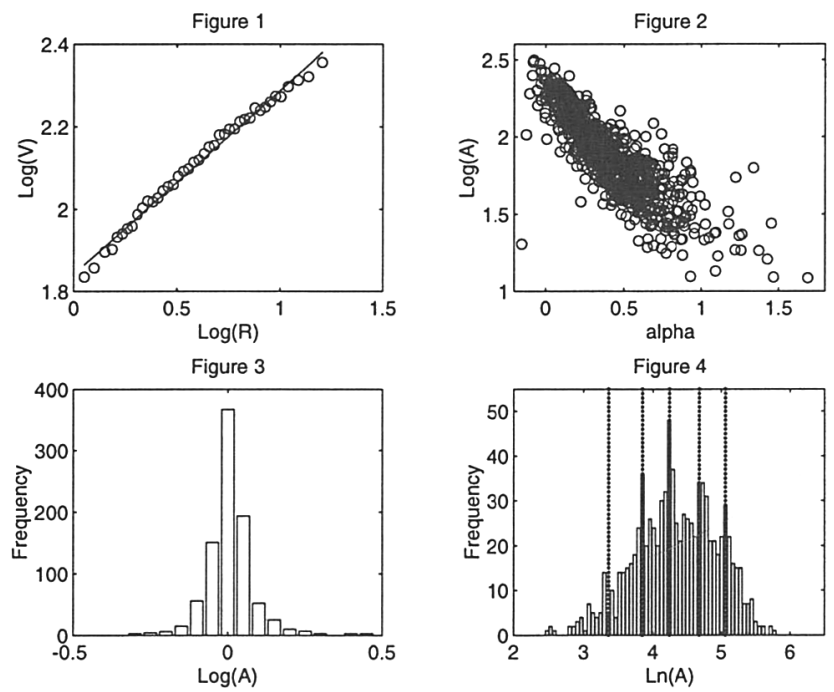

Figure 1. Mean Of 900 Individual Rotation Curves

The results, plotted in Figure 1, provides solid support for the idea that $V=A R^{\alpha}$ is, at the very least, a good working approximation to $H_{\alpha} \mathrm{RC}$ data, and provides the necessary rationale for a detailed investigation of the power-law hypothesis.

Given $V=A R^{\alpha}$, then $\log V=\log A+\alpha \log R$. The parameters $(\alpha, \log A)$ are estimated by regressing $\log V$ on $\log R$ for each of $900 \mathrm{RCs}$ and the results, plotted in Figure 2, demonstrate an extremely strong $(\alpha, \log A)$ correlation. A detailed analysis of the correlation, described in the following, shows it strongly orders the 900 galaxies according to their luminosity properties.

\section{The Regression Model And A Testing Of It}

A detailed analysis shows the best model of Figure 2 to be $\log A=\log V_{0}-$ $\alpha \log R_{0}$ so that

$$
\frac{V}{V_{0}}=\left(\frac{R}{R_{0}}\right)^{\alpha}, \text { where }
$$




$$
\begin{aligned}
& \log V_{0}=-0.584-0.133 M-0.000243 S, \\
& \log R_{0}=-3.291-0.208 M-0.00292 S,
\end{aligned}
$$

and $S$ is surface brightness in solar luminosities per square parsec. This model accounts for over $90 \%$ of the variation in Figure 2.

As a final test of the model, for each galaxy we computed $\left(R_{0}, V_{0}\right)$ according to (5), formed the scaled profile $\left(R / R_{0}, V / V_{0}\right)$ and regressed $\log \left(V / V_{0}\right)$ on $\log \left(R / R_{0}\right)$. Given (5), then the resulting regression constant should be statistically zero. The distribution of the 900 regression constants is shown in Figure 3, for which a $95 \%$ confidence interval for the mean value is computed as $(-0.003,+0.007)$. Thus, for all practical purposes, the regression constant can be considered as statistically zero, thereby confirming the quality of (5).

\section{A Super-TF Relationship}

Evaluating (5) at $R=R_{\text {opt }}$ and inverting, gives $M=f\left(V_{o p t}, R_{o p t}, S, \alpha\right)$. Since (a): $S$ is a function $M$ and $R_{o p t}$; (b): the radial scale of any given galaxy is ultimately determined as a function of its angular scale, its apparent magnitude and its absolute magnitude via the relation $m-M=5 \log d-5$, where $d$ is object distance; (c): $\alpha$ is independent of any linear scale change, and is therefore determined entirely by velocity information; then $M=f\left(V_{\text {opt }}, R_{\text {opt }}, S, \alpha\right)$ can be seen as an implicitly defined non-linear equation for the determination of $M$ given total rotational velocity information. It can therefore be seen as a form of super-TF relation for the determination of absolute magnitudes.

\section{The Discrete Dynamics Hypothesis}

The power-law hypothesis was originally tried against a subsample of the $21 \mathrm{RCs}$ of late-type spirals given in the classical paper of Rubin, Ford \& Thonnard (1980; hereafter RFT); this trial took the form of performing a linear regression of $\ln V_{\text {rot }}$ on $\ln R$ for that subset of twelve RCs which manifested purely monotonic behaviour, and then recording the regression constants. The results of this preliminary exercise, quoted to two significant figures, are condensed in Table 1. The salient feature of this table is that, after taking into account the numerical rounding process, $\ln A$ only takes values which lie within \pm 0.15 of an integer or half-integer.

\begin{tabular}{|lccccccc|}
\hline \multicolumn{7}{|c|}{ Table 1: $V=A R^{\alpha}$} \\
\hline Galaxy & Ln(A) & Galaxy & Ln(A) & Galaxy & Ln(A) & Galaxy & Ln(A) \\
\hline N3672 & 3.6 & U3691 & 3.6 & N3495 & 4.0 & N4605 & 4.0 \\
I0467 & 4.1 & N0701 & 4.1 & N1035 & 4.1 & N4062 & 4.5 \\
N2742 & 4.5 & N4682 & 4.5 & N7541 & 4.6 & N4321 & 4.9 \\
\hline
\end{tabular}

The likelihood of this result is determined as follows: the probability that a single number, chosen at random from the real line, will lie within \pm 0.15 of an integer or half-integer is exactly 0.6 . Consequently, the probability that every one of a sample of twelve numbers, chosen on the basis of an independent prior criterion, lying within \pm 0.15 of an integer or half-integer is $0.6^{12} \approx 0.002$. Thus, 
the RFT data suggests that $\ln A$ might be periodic with period 0.5 and zero phase.

Considering details, we noted how RFT's use of $\mathrm{H}=50 \mathrm{~km} / \mathrm{sec} / \mathrm{Mpc}$ to set linear scales (against today's consensus that $\mathrm{H}>50 \mathrm{~km} / \mathrm{sec} / \mathrm{Mpc}$ by a large margin) implies these linear scales to be too big by a similar margin. Consequently, to make PS data (with its TF-based linear scales) comparable to the RFT data, it was necessary to scale the PS data in some suitable way. This problem was addressed by noting that, if for example, $\mathrm{H}=70 \mathrm{~km} / \mathrm{sec} / \mathrm{Mpc}$ was the correct value of $H$, then RFT linear scales would be too great by a factor of 1.4. Given the actual uncertainty in $H$, this led to the formulation of the specific hypothesis that, when $V=A\left(R / R_{0}\right)^{\alpha}$ is fitted to the PS data, there exists a scaling value, $1 / R_{0}$, somewhere in the range $1.0 \leq 1 / R_{0} \leq 2.0$ which is such that $\ln A$ is distributed with period 0.5 and zero phase.

\section{The Search and Results}

The analysis introduced eleven experimental scalings, $1 / R_{0}=1.0,1.1, \ldots 2.0$ and, at each of these, the specific question posed was how many $\ln A$ values lie within \pm 0.15 of either integer or half-integer values? The results are condensed into Table 2 for two partitions of the data; a 'Hit' is defined to be when a particular $\ln A$ lies within \pm 0.15 of an integer or half-integer.

\begin{tabular}{|ccccl|}
\hline \multicolumn{5}{c|}{ Table 2 } \\
\hline Galaxy & Optimal & Sample & Number & $\begin{array}{l}\text { Single Trial } \\
\text { Types }\end{array}$ \\
$R_{0}$ & Size & Of Hits & Probability \\
\hline \hline 0.5 & $1 / 1.5$ & 485 & 304 & 0.123 \\
$6 . .9$ & $1 / 1.7$ & 415 & 292 & $0.731 \times 10^{-5}$ \\
\hline
\end{tabular}

The final column gives the probability of the recorded result occurring by chance alone assuming the optimal scaling was chosen correctly without searching. Given that, in practice, a search over a range of eleven (adjacent) experimental scalings was executed, then the actual probabilities are at least an order of magnitude less significant and, as is clear from the table, the only significant result occurred for late-type spirals.

The actual probability of obtaining the result of Table 2 for late-type spirals when a search over eleven adjacent experimental scalings was used were assessed using Monte-Carlo simulations based on synthetic data generated from the data of Figure 2. This probability was estimated at $6.2 \times 10^{-5}$ over $2 \times 10^{6}$ simulations.

\section{All Spirals Together}

The analysis only found a positive result for late-type spirals. However, further investigation showed that $\alpha$-values for early-type spirals were significantly noisier than for late-type spirals, and this lack of fidelity was sufficient to degrade any possible result for early-type spirals.

The problem was avoided in the following way: for the late-type spirals, it was found that there existed a direct 1:1 correspondence between the integer/halfinteger peaks in the $R_{0}=1 / 1.7$ rescaled $\ln A$ data, and a set of identifiable peaks 
in the TF-scaled $\ln A$ data. But now it was found that these peaks were exactly mirrored by corresponding peaks in the TF-scaled $\ln A$ data for early-type spirals. Figure 4 shows the distribution of $\ln A$ for all $900 \mathrm{RCs}$ together; the dotted lines define the positions of the $\ln A$ peaks in the late-type spiral data which correspond exactly to the $3.0,3.5,4.0,4.5,5.0$ and 5.5 peaks in the $R_{0}=1 / 1.7$ rescaled data. The effect of combining all the data into Figure 4 has been to dramatically amplify these peaks well above the background noise and, given the hypothesis originally raised on RFT data, the overall probability of this result has been conservatively estimated at about $10^{-7}$.

\section{Conclusions}

The first part of the analysis has shown that, once the effect of the bulge on disc-dynamics has been accounted for then, at the very least, the power-law model must be considered as an extremely good approximation for the kinematical behaviour of an idealized disc (that is, one without the irregularities that inevitably occur in real discs).

The second part of the analysis tested an hypothesis, raised initially on RFT data, that disc-kinematics have a preference for certain discrete states; this hypothesis has been confirmed at an extremely high level of confidence. Given the correlation between the kinematic and luminosity properties of spiral galaxies, this translates into the hypothesis that spiral galaxies exist in discrete luminosity states.

Finally, if (5) is inverted, and applied at the optical radius, then it gives $M=f\left(V_{o p t}, R_{o p t}, \alpha\right)$. Given that $R_{o p t}$ is defined as a function of $M, m$ and the angular size of an object, this latter equation can be seen as a super-TF relationship for the determination of absolute magnitudes given $V_{o p t}$.

\section{References}

Mathewson, D.S., Ford, V.L., Buchhorn, M. 1992 A Southern Sky Survey of the Peculiar Velocities of 1355 Spiral Galaxies Astrophys J. Supp. 81 413-659.

Persic, M., Salucci, P., 1995 Rotation Curves of 967 Spiral Galaxies Astrophys. J. Supp. 99 501-541.

Rubin, V.C., Ford, W.K., Thonnard, N. 1980 Rotational Properties of 21 Sc Galaxies With A Large Range Of Luminosities And Radii From NGC $4605(\mathrm{R}=4 \mathrm{kpc})$ To UGC 2885 ( $\mathrm{R}=122 \mathrm{kpc})$ Astrophys. J. 238 471-487. 